Revue d'histoire du XIXe siècle

Société d'histoire de la révolution de 1848 et des

révolutions du XIXe siècle

49 | 2014

1814-1815. Expériences de la discontinuité

\title{
Écrire la discontinuité, et en mourir : L'Hermite de la Chaussée d'Antin en avril 1814
}

Document présenté par Judith Lyon-Caen

Judith Lyon-Caen

\section{(2) OpenEdition \\ Journals}

Édition électronique

URL : http://journals.openedition.org/rh19/4752

DOI : $10.4000 /$ rh 19.4752

ISSN : $1777-5329$

Éditeur

La Société de 1848

Édition imprimée

Date de publication : 1 décembre 2014

Pagination : 123-135

ISSN : 1265-1354

Référence électronique

Judith Lyon-Caen, «Écrire la discontinuité, et en mourir : L'Hermite de la Chaussée d'Antin en avril 1814 », Revue d'histoire du XIXe siècle [En ligne], 49 | 2014, mis en ligne le 01 décembre 2014 consulté le 12 novembre 2019. URL : http://journals.openedition.org/rh19/4752 ; DOI : 10.4000/ rh19.4752 


\title{
Écrire la discontinuité, et en mourir : L'Hermite de la Chaussée d'Antin en avril 1814
}

\author{
DOCUMENT PRÉSENTÉ PAR JUDITH LYON-CAEN
}

D'août 1811 à la fin du mois d'avril 1814, La Gazette de France, l'un des quatre quotidiens parisiens alors autorisés, accueillit dans son feuilleton du samedi un "bulletin» sur les mœurs parisiennes du temps. Ces bulletins hebdomadaires, signés "L'Hermite de la Chaussée d'Antin", parurent dès le début 1812 en librairie, chez Pillet, sous le nom de leur véritable auteur, Étienne de Jouy (1764-1846). Militaire reconverti dans les lettres, dramaturge et librettiste apprécié, Jouy était entré dans le monde restreint de la presse impériale en devenant en 1807 le censeur du Publiciste, ensuite absorbé par La Gazette. L'Hermite fit la fortune journalistique et éditoriale de Jouy. Quand ses livrets d'opéra, comme celui de La Vestale de Spontini ou du Guillaume Tell de Rossini, furent moins joués, quand ses tragédies en vers furent jugées dépassées, c'est encore à son pittoresque Hermite, cet observateur perspicace de la vie parisienne, que Jouy dut une grande part de sa postérité au XIXe siècle : on saluait en lui l'un des pères de la chronique de presse tout en le tenant à la fois comme un descendant de Louis-Sébastien Mercier et un précurseur du flâneur - un sémiologue urbain avant l'heure.

Vieil excentrique établi dans un faubourg, naguère campagnard, d'un Paris en pleine expansion, l'Hermite de 1811 racontait de petites histoires plaisantes sur les habitudes et les ridicules parisiens et informait sur les activités et les spectacles à la mode, bains à la turque ou montagnes russes. Ses "observations» hebdomadaires brossaient le portrait d'un destinataire avide de toutes les nouveautés possibles - travaux sur la voie publique ou couleur des rubans en vogue sur les chapeaux féminins -, mais incrédule en matière politique. Jouy avait ainsi introduit dans la presse cadenassée de l'Empire un semblant d'actualité vivante et colorée, faite de bavardages et de rumeurs, où l'on croisait toutes sortes de personnages, des artistes, des cochers de fiacre, des femmes à la mode, des rentiers, des commerçants... Mais, à moins de savoir lire entre les lignes, on ne parlait pas de politique dans le feuilleton de la Gazette de France et les "observations morales» réunies dans les jolis petits volumes in-12 du libraire Pillet appelaient encore moins à une lecture contextualisée. Pourtant, L'Hermite de la Chaussée d'Antin meurt dans 
La Gazette de France en avril 1814, et c'est sur cette mort que s'achève la série publiée chez Pillet à quelques semaines d'écart. Quel sens pouvait avoir, en avril 1814, une observation des mœurs détachée des enjeux politiques du présent? Et comment Jouy, journaliste au service de l'Empire, pouvait-il continuer à écrire alors que les conditions de l'exercice de la presse se trouvaient bouleversées?

On a voulu ici donner à lire les derniers bulletins de l'Hermite de la Chaussée d'Antin, qui sont aussi la chronique de ses derniers moments... Car Jouy a suivi la rupture d'avril 1814 depuis le feuilleton de la Gazette de France, en regardant se défaire sous ses yeux le système politique et administratif qui rendait possible son écriture de journaliste mais en délimitait strictement l'aire et le registre. Et il a décidé de faire mourir son Hermite au moment même où il ne pouvait plus continuer, comme auteur, à le faire vivre. Les bulletins que l'on va lire sont donc, exemplairement, une écriture de la discontinuité, une écriture aux prises avec la rupture politique (l'entrée des armées étrangères dans Paris, la restauration du pouvoir monarchique), et une écriture tentant de raconter et de figurer la discontinuité la plus radicale dans un langage entièrement constitué pour commenter sur un ton badin le flux continu de la vie parisienne. L'originalité de Jouy tient à ce choix de ne pas quitter le journal en catimini (en interrompant la publication) mais de tenir jusqu'au bout - pour renaître ailleurs, on va le voir. Etait-ce panache de la part de Jouy, qui, en homme de théâtre, tenait à faire mourir son personnage sur scène? Faut-il y voir le souci de rester dans la place jusqu'au dernier moment, pour ménager les possibles? Aucun autre écrit ne permet d'éclairer le choix de Jouy. Mais la lecture de ses bulletins fait éprouver l'action d'écriture du journaliste, qui, à l'abri de son personnage, décrit prudemment ce qu'il voit du glissement de la souveraineté, dans ces semaines du début d'avril 1814 où le projet de constitution hâtivement préparé par le Sénat est âprement discuté, et où les modalités du rétablissement des Bourbons sur le trône demeurent incertaines.

L'Hermite voit donc l'entrée des armées étrangères dans Paris, et tombe malade : «Les secousses sont fatales à mon âge»... Aveu ironique, qui prélude au déclin rapide et à la mort du vieux bonhomme. En lisant ces chroniques du printemps 1814 , on se confronte à la rupture politique dans le rythme d'une écriture qui doit s'ajuster, semaine après semaine, à une situation mouvante. Cette lecture donne aussi accès, mieux que tout commentaire, à une pratique scripturaire de l'équivoque qui, en ces temps de trouble, se cultive comme une précieuse compétence. Jouy est passé d'une figuration inoffensive de la diversité du monde social à une discrète, et d'autant plus efficace, écriture politique polyphonique. La fiction des derniers moments de l'Hermite entremêle en effet toutes les ambiguïtés et tous les sous-entendus possibles. L'Hermite exulte en voyant l'entrée à Paris du nouveau roi, mais doit garder la chambre : son âme vit dans l'allégresse, mais son corps meurt, alors que 


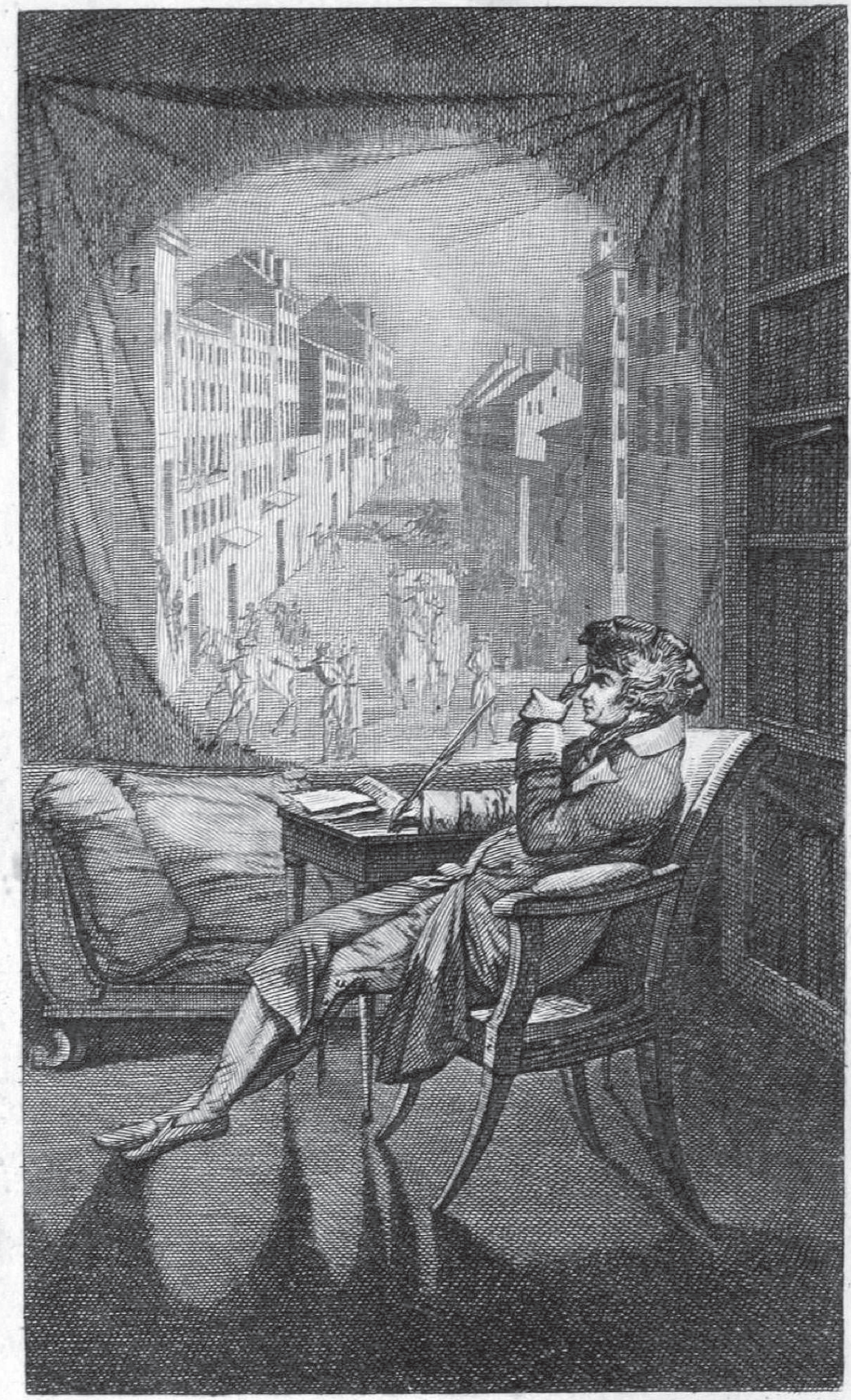

\footnotetext{
e fle Citute off comme une Chambre obscure

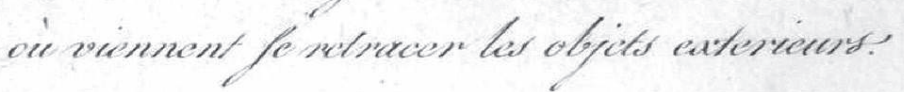


son médecin assure qu'il n'est point malade et "qu'on ne meurt que lorsqu'on le veut bien". À mesure que sa fin approche, l'Hermite se sent d'ailleurs d'une liberté d'esprit croissante - et la parole suit : l'empereur est devenu Bonaparte, et le vieil homme, dans ses derniers instants, boit au «bon roi Louis XVIII et à la paix du monde", non sans avoir appelé le nouveau régime à garantir la liberté publique et l'indépendance des tribunaux. Mais le personnage n'est pas l'auteur : Jouy aurait pu, en cas de besoin, rappeler que son Hermite de fiction avait perdu un peu la tête. L'ambiguïté sature l'écriture, probablement au delà même de ce que notre lecture peut en déceler. Ainsi de cette affirmation apparemment anodine, «le cercle révolutionnaire est achevé», que l'Hermite assortit immédiatement de l'évocation des «bienfaits d'une constitution " : le retour en arrière ne mène pas en 1789, mais plutôt à l'instauration de la monarchie constitutionnelle en 1791. Ainsi, également, des sous-entendus ironiques : le vieil Hermite est aussi malade que le roi luimême, âgé et accablé de goutte. La maladie n'est pas dite, mais évoquée par le biais du souvenir de l'entrée royale de Louis XV "après la maladie de Metz" en 1744. L'Hermite serait-il malade de cette restauration si mal portante?

La publication des bulletins hebdomadaires de L'Hermite de la Chaussée d'Antin s'acheva le 30 avril 1814, avec un ultime feuilleton intitulé "Le testament de l'Hermite" qui, fort bavard, n'est pas reproduit ici. Dès le 7 mai, les lecteurs de la Gazette de France firent la connaissance d'un nouveau personnage, "Guillaume le Franc Parleur», qui continua l'œuvre de l'Hermite jusqu'en novembre 1814. Jouy et son Guillaume quittèrent alors la Gazette de France pour le Journal général où ils restèrent jusqu'à fin mars 1815. Avec les Cent-Jours, Jouy reprit son service à la Gazette, où il célébra le retour de l'Empereur aux Tuileries avant un ultime réajustement, le 8 juillet, où Guillaume délivra sa "profession de foi politique», patriote et monarchiste. "Le Franc-Parleur" avait tout de même survécu à bien des revirements ${ }^{1} \ldots$ En juillet 1815, dans Le Mercure de France, Jouy mit son Guillaume à la retraite et convoqua un deuxième Hermite, ami d'enfance du premier, le chevalier de Pageville, un vieillard octogénaire tout juste revenu de la Guyane, où il s'était établi «avant que la révolution commençât». Et c'est avec cet Hermite de la Guyane ${ }^{2}$ opportunément vierge de tout passé politique que Jouy, désormais

1. Guillaume le Franc Parleur, ou Observations sur les mours françaises au commencement du $X I X^{e}$ siècle, Paris, Pillet, 2 volumes, 1815-1817. Publiée sous la seconde Restauration, cette édition diffère légèrement des feuilletons publiés dans la presse pendant les Cent-Jours. Mais après quatre éditions parisiennes de Guillaume, Jouy a également fait publier en 1818 une édition imprimée à Bruxelles conforme aux originaux. On y lit notamment le feuilleton du $1^{\text {er }}$ avril 1815 dans lequel Guillaume critique vertement les premiers actes du gouvernement de la Restauration, comme «autant de manifestes contre les idées et les principes libéraux dont la nation était décidée à ne point se départir".

2. L'Hermite de la Guiane, Observations sur les moeurs et les usages français au commencement du $X I X^{e}$ siècle, Paris, Pillet, 3 volumes, 1816-1817. Jouy publia ensuite, avec des collaborateurs, une série intitulée L'Hermite en province (Paris, Pillet, 1819-1820), puis, avec Jay, des «Hermites» libéraux : Les Hermites en prison, ou Consolations de Sainte-Pélagie, Paris, Ladvocat, 1823, 2 volumes, et Les Hermites en liberté, Paris, Ladvocat, 1824, 3 volumes. 
durablement identifié à l'opposition libérale, poursuivit l'entreprise d'observation des «mœurs nationales» initiée sous l'Empire.

*

9 avril 1814

\section{Prise de Paris}

[...] Au nombre des événements que tant de secousses politiques ont pu faire craindre, celui de l'occupation de la capitale par des armées étrangères n'est jamais entré dans mon esprit. J'avais pour garant de ma sécurité treize siècles d'une possession vierge; car je persiste à ne point voir une conquête dans la prise de Paris, sous le règne de Charles VI : les Anglais y furent appelés, introduits et maintenus par les factions, par la démence du Roi, par la perfidie de la reine et par la proscription du Dauphin. Les autres sièges de Paris appartiennent à l'histoire de nos discordes civiles, et sont tout à fait étrangers au succès de nos armées ennemies.

Il était aisé de prévoir que la France, poussée hors de toutes limites, débordée comme un torrent sur l'Europe entière, épuisée par d'innombrables sacrifices, écrasée par ses conquêtes, dégoûtée de la guerre, et même de la gloire; il était, dis-je, aisé de prévoir que la France était menacée d'une grande catastrophe.

L'Europe s'est liguée contre l'oppression; ses armées coalisées sont venues conquérir une paix vainement et si longtemps invoquée : la sainteté de leur cause a doublé leur nombre et justifié leur succès : quinze mois ont suffi pour ramener nos légions des bords de la Moskowa aux rives de la Seine.

De tous les spectacles qu'on pouvait offrir aux Parisiens, le plus nouveau, comme le plus terrible, était celui d'une bataille. Depuis plus de deux siècles, la guerre n'avait point approché de leurs murs; le bruit des armes ne retentissait depuis longtemps à leurs oreilles que dans les marches triomphales; et leurs femmes pouvaient dire comme celles de Spartiates, quelles n'avaient jamais vu la fumée du camp ennemi: l'orage grondait sur leur tête, les Parisiens se croyaient à l'abri de la foudre. Un gouvernement fallacieux entrete-

3. Affublé de quatre girouettes par le Dictionnaire des girouettes publié «par une société de contemporains " à la fin de 1815 (Paris, Alexis Eymery), Jouy a lui-même participé aux entreprises de mise en ordre biographique de l'époque en publiant entre 1820 et 1825, avec Arnault, Jay et Norvins, une Biographie nouvelle des contemporains ou Dictionnaire historique et raisonné de tous les hommes qui, depuis la Révolution Française, ont acquis de la célébrité par leurs actions, leurs écrits, leurs erreurs ou leurs crimes, soit en France, soit dans les pays étrangers..., Paris, Émile Babeuf, 20 volumes. Cf. JeanLuc Chappey, Ordres et désordres biographiques. Dictionnaires, listes de noms, réputation, des Lumières à Wikipédia, Seyssel, Champ Vallon, 2013 et Judith Lyon-Caen, "Étienne de Jouy», in Dominique Kalifa, Philippe Régnier, Marie-Ève Thérenty, Alain Vaillant [dir.], La Civilisation du journal. Histoire culturelle et littéraire de la presse française au XIXe siècle, Paris, Nouveau monde éditions, 2011, p. 11011105. 
nait par tous les moyens possibles cette dangereuse sécurité, et l'ennemi était à nos portes, que les Bulletins nous parlaient encore de victoires.

Les yeux ne commencèrent à s'ouvrir que dans la matinée du 28 mars, à la vue des scènes déchirantes dont les boulevards étaient le principal théâtre : ces paisibles remparts, naguère embellis d'équipages brillants, de femmes élégantes, de tout le cortège, du luxe et des plaisirs, étaient en ce moment couverts de soldats blessés, de villageois abandonnant leur ferme ou leur chaumière, et traînant avec eux les derniers débris de leur chétive fortune : ici, des charrettes où quelques bottes de foin et de paille servaient de lit à des familles entières; là des troupeaux de moutons, des vaches que conduisaient, sur son ânon, leur maître expatrié; plus loin des groupes de citadins effrayés accablant de questions des malheureux qui semblaient soulagés en racontant leur désastre. Que d'épisodes touchants dans ce triste tableau! Que d'exemples de pitié! que d'actions généreuses, que de secours, de consolations, j'ai vu prodiguer par nos bons Parisiens à leurs malheureux compatriotes!

Dès midi, le tableau change, et tout ce qui se passe sur les boulevards n'est plus qu'un spectacle pour la foule qui s'y promène. La confiance semble renaittre; tout prend une attitude guerrière; quelques fuyards, un plus grand nombre de blessés arrive; mais des troupes nouvelles, des munitions, partent en bon ordre; quelques officiers d'ordonnance, en traversant Paris, y sèment des rapports mensongers, et le peuple, non seulement voit sans émotion les mêmes objets qui le glaçaient de crainte quelques heures auparavant, mais il finit par prendre part aux jeux des grimaciers, des charlatans, des marionnettes, sur la même place où il vient de s'entretenir avec terreur du péril imminent dont il est menacé. Les mêmes inquiétudes se renouvellent le lendemain; les mêmes causes les font disparaître.

La postérité se refusera sans doute à croire ou du moins à comprendre, qu'une armée de deux cent mille hommes soit arrivée à deux lieues de cette immense capitale sans que ses habitants en fussent autrement instruits que par le bruit du canon et de la générale que l'on battit le 30 mars, à quatre heures du matin, dans tous les quartiers de la ville.

A ce signal, je sors d'un lit où je ne dormais pas; mes préparatifs avaient été faits la veille; j'endosse un vieil habit de ratine bleue, qui ne ressemblait pas mal à un uniforme; je charge mon épaule d'un fusil de Pauly $y^{4}$, je couvre mon chef d'un bonnet fourré à la polonaise, et dans cet attirail, je me mets en campagne; l'effroi était à son comble dans tous les quartiers de cette vaste capitale; le tambour appelait la garde nationale à défendre une ville qui ne pouvait ni ne devait être défendue; partout des femmes, des enfants en pleurs cherchaient à retenir leurs époux, leurs pères qui s'arrachaient avec effort de leurs bras. Le champ de bataille était pour ainsi dire à ma porte; je m'acheminai vers les hauteurs de Montmartre.

4. Le fusil mis au point par Pauly pour la Grande armée en 1812. 
Poursuivant un odieux système de mensonge et de perfidie, le gouvernement avait annoncé la veille qu'il ne s'agissait que de repousser une faible colonne de l'armée ennemie, et deux cent mille hommes étaient sous nos murs! des masses d'infanterie s'avançaient sur toutes les routes, une cavalerie innombrable couvrait les plaines, 600 pièces d'artillerie foudroyaient les hauteurs!

Aucune mesure n'avait été prise pour repousser une pareille attaque : quelques pièces de canon servies par de courageux enfants, et placées au hasard sur les collines environnantes; douze mille hommes de troupes de ligne, un pareil nombre de gardes nationaux, sans chefs et sans munitions; une ligne de palissades mal disposées, mal jointes, tels étaient nos moyens de défense. Pouvaient-ils avoir été pris dans une autre intention que d'attirer sur cette ville tous les malheurs d'un siège, en lui donnant un aspect guerrier propre à justifier toutes les mesures que pourraient prendre les vainqueurs et tous les excès auxquels ils pourraient se porter?

Après une défense de douze heures contre des forces décuples; lorsque tout paraissait perdu, fors l'honneur; pendant que l'on placardait encore sur les murs une proclamation dans laquelle un roi qui venait de fuir disait : $\mathrm{Je}$ reste avec vous; lorsqu'il ne restait plus qu'à franchir une frêle barrière, objet de dérision pour les Parisiens eux-mêmes; on a vu (chose incroyable) l'armée victorieuse des puissances alliées s'arrêter comme par enchantement aux portes de cette capitale de la France, terme de tant de vœux, de fatigue et de travaux; on a vu des monarques, animés du ressentiment de tant d'outrages, s'interdire l'entrée dans Paris que leur livrait la victoire, et signer, avec un général français, une capitulation, monument de magnanimité dont l'histoire n'offre aucun modèle.

Cette nuit du 30 mars, qui dut être pour Paris une nuit de ravage et de destruction, a vu finir quinze ans de servitude; elle a préparé dans la capitale des arts l'alliance des grandes puissances de l'Europe et la restauration du trône antique et sacré de nos rois légitimes : révolution prodigieuse, que le génie le plus entreprenant n'imaginait plus que dans ses rêves, et qui fut exécutée au moment où l'on put l'entrevoir.

La France, le 30 mars, gémissait sous le joug de Bonaparte; le 31, elle était libre, et appelait Louis XVIII.

Dès la pointe du jour, les boulevards, que devait suivre l'armée des alliés entrant à Paris, étaient, en quelque sorte, inondés des flots d'une population immense : les fenêtres de toutes les maisons étaient encombrées de spectateurs. Quelques patrouilles de la garde nationale suffisaient pour maintenir l'ordre parmi cette multitude de citoyens animés du même esprit et pleins des mêmes sentiments.

Je ne le cache pas, cet appareil nouveau, ces légions accourues des bords du Volga, de la Sprée et du Danube, cette pompe étrangère de la victoire, affligèrent mon cœur; mes yeux se remplirent de larmes; mais l'amour de 
la patrie et de l'humanité l'emportèrent bientôt sur le sentiment de l'orgueil national, et je contemplai avec admiration le spectacle inconnu jusqu'ici d'un monarque étranger reçu comme un bienfaiteur dans la capitale d'un Etat conquis et délivré par ses armes, recueillant avec la plus touchante modestie les hommages dont on l'environne, et répondant aux acclamations d'un peuple ivre de reconnaissance par la délivrance de deux cent mille prisonniers français que le sort de la guerre a fait tomber entre ses mains.

\section{6 avril 1814}

\section{La maladie de l'Hermite}

Les secousses sont fatales à mon âge : il faut un tremblement de terre pour renverser un bâtiment neuf; une détonation un peu forte suffit pour faire crouler une masure. L'économie animale est soumise aux mêmes lois que l'économie domestique; le pauvre et le vieillard doivent également ménager le peu qu'ils possèdent.

Le ciel m'a départi d'assez longs jours, et cependant j'ai peine à concevoir qu'ils aient pu suffire aux événements qui se sont pour ainsi dire accumulés dans l'espace de temps que ma vie embrasse. Les derniers dont je viens d'être témoin, et auxquels mon esprit et mon cœur ont pris une part trop active, ont épuisé mes forces; je me sens attaqué de cette maladie que Fontenelle définit : une difficulté de vivre, et j'ai le pressentiment que j'irai bientôt aider Rabelais dans la recherche du grand peut-être.

Ce que j'ai de mieux à faire dans un moment où toute espèce d'occupation m'est interdite, c'est de me ressasser mes souvenirs, bien sûr de n'y trouver que des consolations pour le présent qui m'échappe, et des espérances pour un avenir dont j'ai le bonheur de ne pas douter.

Le premier événement public que je retrouve gravé dans ma mémoire (moins par la sensation que j'étais alors trop jeune pour éprouver, que par le récit qui m'en a tant de fois été fait), c'est le retour de Louis XV après sa maladie de Metz. Cette entrée d'un monarque bien-aimé, sur les jours duquel on avait conçu de si vives alarmes, n'avait rien d'une pompe triomphale; l'ivresse populaire en faisait tous les frais; c'était une véritable fête de famille, semblable à celle dont cette capitale offrait, mardi dernier, l'image, à l'aspect du petit-fils de Louis XV, rentrant dans Paris après vingt-cinq ans d'absence.

Depuis Lawfeld et Raucoux, la France avait perdu l'attitude de la victoire; la glorieuse journée de Fontenoy produisit un enthousiasme difficile à décrire; et ces deux grandes époques, sur lesquelles s'appuient mes premiers souvenirs, ont fortifié dans mon cœur cet amour pour le noble sang des Bourbons, qui fait partie du caractère national, et que n'ont pu altérer ni le tems ni l'infortune. 
Louis XVI, dans un règne trop court pour le bonheur et pour l'honneur du peuple français, déploya des vertus dont le ciel s'est réservé la récompense. La chute du trône amena les désastres révolutionnaires; l'état penchait vers sa ruine, tous les liens de la société étaient rompus, la nation sentait le besoin d'un chef : l'audacieux Buonaparte se saisit du pouvoir et nous sauva de l'anarchie par la servitude. Le mouvement de la guerre, l'ivresse de la victoire, nous dérobèrent quelque tems la vue de nos fers, et nous gémissions sans honte sous un joug intolérable. Au premier revers on vit chanceler l'édifice immense qu'il avait élevé hors de toutes proportions, et bientôt il se brisa luimême, en tombant du haut de sa prodigieuse fortune.

Le cercle révolutionnaire est achevé; nous nous retrouvons au point d'où nous sommes partis. Puisse cette longue et sanglante leçon reçue par les pères ne pas être perdue pour les enfants! Que les nôtres apprennent par notre expérience à quel prix s'achètent les institutions nouvelles, et qu'ils jouissent avec reconnaissance, au sein de la monarchie, des bienfaits d'une constitution qui peut seule maintenant en garantir la durée.

On peut en croire le fougueux cardinal de Retz sur les dangers de discuter les lois établies. Voici comment il peint une de ces assemblées tumultueuses de la Fronde : «on chercha, en s'éveillant, comme à tâtons, les lois; on ne les trouva plus; on s'effara, l'on cria, l'on se les demanda, et dans cette agitation, les questions que leurs explications firent naître, d'obscures qu'elles étaient et vénérables par leur obscurité même, devinrent problématiques; et là, à l'égard de la moitié du monde, odieuses; le peuple entra dans le sanctuaire : il leva le voile qui doit couvrir l'origine d'où sont émanés les droits des peuples, et ceux des rois, qui ne s'accordent jamais mieux ensemble, que dans l'ombre et le silence.» Nous avons appris à nos dépens et au profit de nos neveux, qu'il était plus facile de nier ces principes que d'en éviter les conséquences.

Je m'enfonçais de plus en plus dans les profondeurs de la politique; j'en sortis par ordonnance du médecin. C'est un original que mon ami le docteur $\mathrm{N}^{* * *}$. En dépit de la fièvre qui me galopait, il me prouva que je n'étais point malade, et finit par m'assurer, le plus sérieusement du monde, qu'on ne meurt que lorsqu'on le veut bien. Je lui objectai que cette volonté-là vient toujours avec l'âge.

- Il n'y a point d'âge, continua-t-il ; la vieillesse est un vieux préjugé, et la santé du corps, accident à part, dépend de celle de l'âme.

- Dans ce cas-là, docteur, comment expliquerez-vous ma maladie, quand je vous aurai donné l'assurance que mon âme ne s'est jamais mieux portée! Je suis né sous les Bourbons; je me retrouve avec bonheur sous leur empire; tous mes vœux, de ce côté du tombeau, sont à peu près comblés; je me rattache à la vie tant que je peux, et quoi que vous en puissiez dire, je sens qu'elle m'échappe.

- C'est que vos réflexions ne sont pas toutes d'accord avec vos sentiments; c'est que votre esprit est contristé par des observations chagrinantes, 
en même tems que votre cœur nage dans la joie : il résulte de ce conflit de sensations et de pensées un état violent que vous appelez maladie, et qu'il dépend de vous de faire cesser.

- Docteur, il y a du vrai dans ce que vous dites : je jouis délicieusement du bonheur de ma patrie; je revois avec transport le chef d'une maison adorée remonter au trône de ces ancêtres; mais si je détourne un moment les yeux de ce tableau sublime, je vois l'intrigue aux cent pieds, aux cent bras, qui déjà s'empare de toutes les avenues du pouvoir; je vois la bassesse encore souillée de la fange où elle se traînait la veille, profaner aujourd'hui l'éloge, en se hâtant de s'en rendre l'interprète; j'entends prodiguer la menace et l'outrage à l'ennemi qui n'est plus à craindre, et je vois une foule de braves le lendemain de la victoire.

- Eh! Mon pauvre Hermite, c'est bien la peine d'avoir vécu 75 ans pour s'étonner de pareilles choses! Le navire est à flot, les vents sont bons, le pilote est au gouvernail; est-ce le tems de penser aux souris qui rognent la cargaison? Plus d'humeur; éloignez toutes les pensées tristes; prenez mon bras, et venez à quelques pas d'ici jouir du spectacle ravissant d'un petit-fils de notre Henri IV, reçu par des sujets auxquels il apporte, comme son aïeul, la paix, le bonheur et le généreux oubli de toutes les fautes, de toutes les erreurs.

Le docteur me pressa; je fis un effort pour passer un habit; ma faiblesse trahit mon courage. J'allais y renoncer; une musique militaire se fit entendre sous mes fenêtres : je prête l'oreille; j'entends l'air national de vive Henri $I V$ ! Mes forces renaissent; je m'habille, et soutenu par le docteur et par mon fidèle Paul, je parvins à me transporter sur le passage de Monsieur.

Quelles délicieuses émotions j'éprouvai en revoyant ce prince d'un caractère si franc, si loyal, si français; sa figure, où l'on aimait à reconnaître quelques traits du bon Henri, rayonnait de joie et de bonheur. Tout était français dans sa personne; sa grâce, ses manières, cette expression d'amour et de confiance qui caractérise sa noble race, cet habit national, et ce panache national aussi, puisque c'est celui du Béarnais. Dans ce moment, je me sentis renaître : un cri s'échappa de ma bouche et de mon cœur : Vive le Roi! Ce mot, que j'avais bégayé dans mon enfance, à l'entrée de Louis XV, j'ai donc pu le répéter soixante-dix ans après! J'ai pu voir, après tant d'orages, la nation rendue à son antique loyauté, se rallier comme une famille autour d'un père chéri; j'aurai vu les factions s'éteindre, tous les cœurs se réunir dans l'intérêt de la patrie, et toutes les volontés se confondre dans le vœu du bonheur public, fondé sur la double base de l'amour du prince et du respect des lois. La nature peut disposer de moi; j'ai assez vécu.

Depuis ce jour d'éternelle mémoire, je suis confiné dans mon lit; je crains bien, malgré ma bonne volonté, de ne plus sortir moi-même. S'il en est ainsi, un seul regret viendra troubler mes derniers moments; c'est de n'avoir pu contempler avant de mourir cette auguste princesse, exemple de piété filiale, modèle de toutes les vertus, à qui les Français doivent tant d'amour 
en dédommagement de tant de malheurs, et qui n'a pu quitter sans regrets la patrie où ses yeux ne s'étaient ouverts que pour répandre des larmes.

\section{3 avril 1814}

\section{La Mort de l'Hermite}

Le moment est venu; je sens que je n'achèverai pas la journée qui commence, et je profite d'un mouvement de fièvre, qui rend à mon sang et à mon esprit quelqu'activité, pour laisser tomber sur le papier les dernières lignes que tracera ma main défaillante. [...]

Vendredi, 22 avril 1814

L'Hermite de la Chaussée d'Antin a cessé de vivre; il s'est endormi dans un sommeil éternel hier à quatre heures du soir, à l'âge de soixante-treize ans, deux mois et quelques jours. Puisque l'Hermite est devenu, par accident, un personnage public, et que ses Discours ont eu quelque succès dans le monde, j'ai pensé qu'il était de mon devoir, en ma qualité de parent et d'exécuteur testamentaire, de rendre compte à ses amis (au nombre desquels il se plaisait à compter ses lecteurs), les derniers moments d'un grand oncle dont j'ai tant de raisons de chérir et d'honorer la mémoire. J'ai pu croire, d'ailleurs, que ces détails, où l'on reconnaît les traces de son caractère observateur, ne seraient point déplacés à la suite de ses Observations sur les mœurs.

J'avais été moins alarmé que je n'aurais dû l'être d'une maladie dont mon oncle avait lui-même informé le public, et sur les suites de laquelle les rapports du médecin et mes propres observations me rassuraient également. L'Hermite parlait de sa fin prochaine avec une si grande liberté d'esprit, quelquefois même avec tant de gaieté; je remarquais si peu d'altération dans ses traits, si peu d'affaissement dans ses forces physiques et morales, que je m’obstinais à ne voir, dans l'idée qui le préoccupait exclusivement, que le texte d'un de ses prochains Discours.

Ce ne fut que dimanche matin, en trouvant au chevet de son lit un notaire auquel il dictait ses dernières volontés, que je commençai à concevoir des inquiétudes dont je ne fus pas le maître de lui dérober la vive impression.

"Mon cher Ernest (me dit-il, avec un sourire plein de douceur), vous êtes surpris de tout, parce que vous ne vous préparez à rien [...]. Mourir est une des clauses du contrat de la vie; et j'ai bien fait d'attendre un peu tard pour la remplir, puisque mes yeux, avant de se fermer, ont vu luire sur la France l'aurore d'un jour qui semblait ne devoir jamais naître, ou du moins ne devoir jamais se lever pour moi. Si la nature m’avait laissé le choix du moment où je devais lui payer ma dette, aurais-je pu en saisir un meilleur? J'ai vu, contre toute vraisemblance, s'accomplir le grand événement d'une restauration qui prépare à ma patrie de nouveaux siècle de prospérité; je 
jouis dès à présent de tous les biens qui vous sont réservés, avec la certitude de n'être pas témoin des derniers efforts que la sottise, l'orgueil et l'intrigue mettront en œuvre pour retarder l'établissement d'un ordre de choses où le mérite et la probité seront les seuls titres à l'estime de la nation et à la faveur du prince. J'admire, en ma qualité d'homme, l'exemple unique de la magnanimité qu'un Alexandre, le véritable grand Alexandre, vient de donner au Monde, et je n'aurai point à gémir, comme Français, d'un événement dont la gloire est étrangère à mon pays, dont les suites les plus immédiates ne seront peut-être pas sans amertume, et dont les avantages seront nécessairement le fruit de plus d'un sacrifice.»

Le docteur arriva au moment où mon oncle, échauffé par ce qu'il appelait son esprit prophétique, commençait son discours de prédications : il imposa silence au malade, et le força de donner quelque repos à son corps, en laissant reposer sa tête. [...]

Le lendemain matin, quelque diligence que j'eusse faite, j'avais été devancé auprès de mon oncle par Mme $\mathrm{L}^{* * *}$, son amie la plus intime, dont il a souvent été question dans ses Discours: sa présence semblait l'avoir ranimé; l'espérance me revint.

La matinée fut calme : l'Hermite reçut plusieurs visites, se fit lire les journaux, et provoqua lui-même une discussion sur les affaires publiques, qui l'occupaient exclusivement depuis sa maladie.

"On peut me croire, disait-il, mes opinions sont aujourd'hui bien désintéressées; mes vœux ne sont plus que des espérances. Il n'y a de repos, de bonheur possibles pour la France, qu'au sein de cette monarchie constitutionnelle que Montesquieu préconise avec tant d'éloquence, et dont une nation voisine s'est chargée de nous prouver les avantages. » Le chevalier de $\mathrm{N}^{* * *}$ se récria contre cette proposition, et parla en faveur de la monarchie pure, c'est-à-dire, absolue, du ton d'un homme qui récite ses principes quand il défend ses préjugés. "Eh, M. le chevalier, lui répondit l'Hermite, pour Dieu! ne soyez plus plus royaliste que le Roi; c'est lui-même qui vous en prie. Vous aurez beau dire et beau faire, le siècle va son train; il faut marcher avec lui, et vous ne ferez plus accroire à personne que, même sous un bon prince, le despotisme ne soit pas le pire de tous les gouvernements. Plus les Français chérissent ces Bourbons que la bonté du ciel vient de leur rendre, plus ils doivent mettre leur trône à l'abri des secousses qui l'ont renversé : cet abri, ils ne peuvent le trouver que dans un état de choses qui identifie en quelque sorte la nation avec celui qui la gouverne, qui affermit l'autorité royale, et garantit la liberté publique; qui met hors d'atteinte l'indépendance des tribunaux, et qui consacre en même temps la responsabilité des ministres et l'inviolabilité du monarque. Maintenez, surtout, maintenez, avec des restrictions légales, cette liberté de la presse dont l'utilité est suffisamment démontrée par les soins que Bonaparte avait pris pour la proscrire. Du jour où il put être sûr qu'aucun livre ne paraitrait que dégradé, mutilé par la censure, l'avilisse- 
ment de la nation fut au comble, et la tyrannie ne connut plus de bornes : de là ce déluge d'absurdités, de mensonges et de turpitudes dont la France fut inondée pendant 10 ans, et qui n'accusait pas moins l'imbécile crédulité du peuple que l'impudence du gouvernement. On pouvait appliquer à son chef ce mot de don Luis De Haro, ambassadeur d'Espagne aux conférences des Pyrénées, à qui l'on demandait ce qu'il pensait du cardinal Mazarin : c'est un grand homme, disait-il, mais il a un grand défaut : c'est de vouloir toujours tromper.»

Je voyais que mon oncle se fatiguait beaucoup en parlant : Mme de $\mathrm{L}^{* * *}$ me faisait signe d'emmener deux ou trois interlocuteurs qui nourrissaient impitoyablement la dispute; j'avais besoin, pour y réussir, que le docteur vint à mon aide. Il entra, il salua tout le monde avec un sérieux hippocratique, s'approcha du lit du malade, lui tâta le pouls, se recueillit un moment, prit une prise de tabac, et congédia poliment l'assemblée, à l'exception de Mme de $\mathrm{L}^{* * *}$; du chevalier, de moi et du docteur lui-même, que le malade retint à dîner. [...]

Sans écouter les remontrances du docteur, [mon oncle] donna l'ordre qu'on dressât la table auprès de son lit; et pendant le repas, où il se montra plus fort que nous ne l'avions vu depuis longtemps, il ne fut question que de l'événement miraculeux de la restauration. Le bon Hermite but un verre de vin de Bourgogne à la santé de Louis XVIII et à la paix du monde. [...] 